\title{
Syntheses, Structures and Photoluminescence Property of Metal(II) Halide Complexes with Pyridine-Containing Flexible Tripodal Ligands
}

Gang Wu, ${ }^{\dagger}$ Xiao-Feng Wang, ${ }^{\dagger}$ Taka-aki Okamura, ${ }^{\dagger}$ Wei-Yin Sun, ${ }^{*},{ }^{\dagger}$ and Norikazu Ueyama

Coordination Chemistry Institute, State Key Laboratory of Coordination Chemistry, School of Chemistry and Chemical Engineering, Nanjing University, Nanjing 210093, China, and Department of Macromolecular Science, Graduate School of Science, Osaka University, Toyonaka, Osaka 560-0043, Japan

Supporting Information

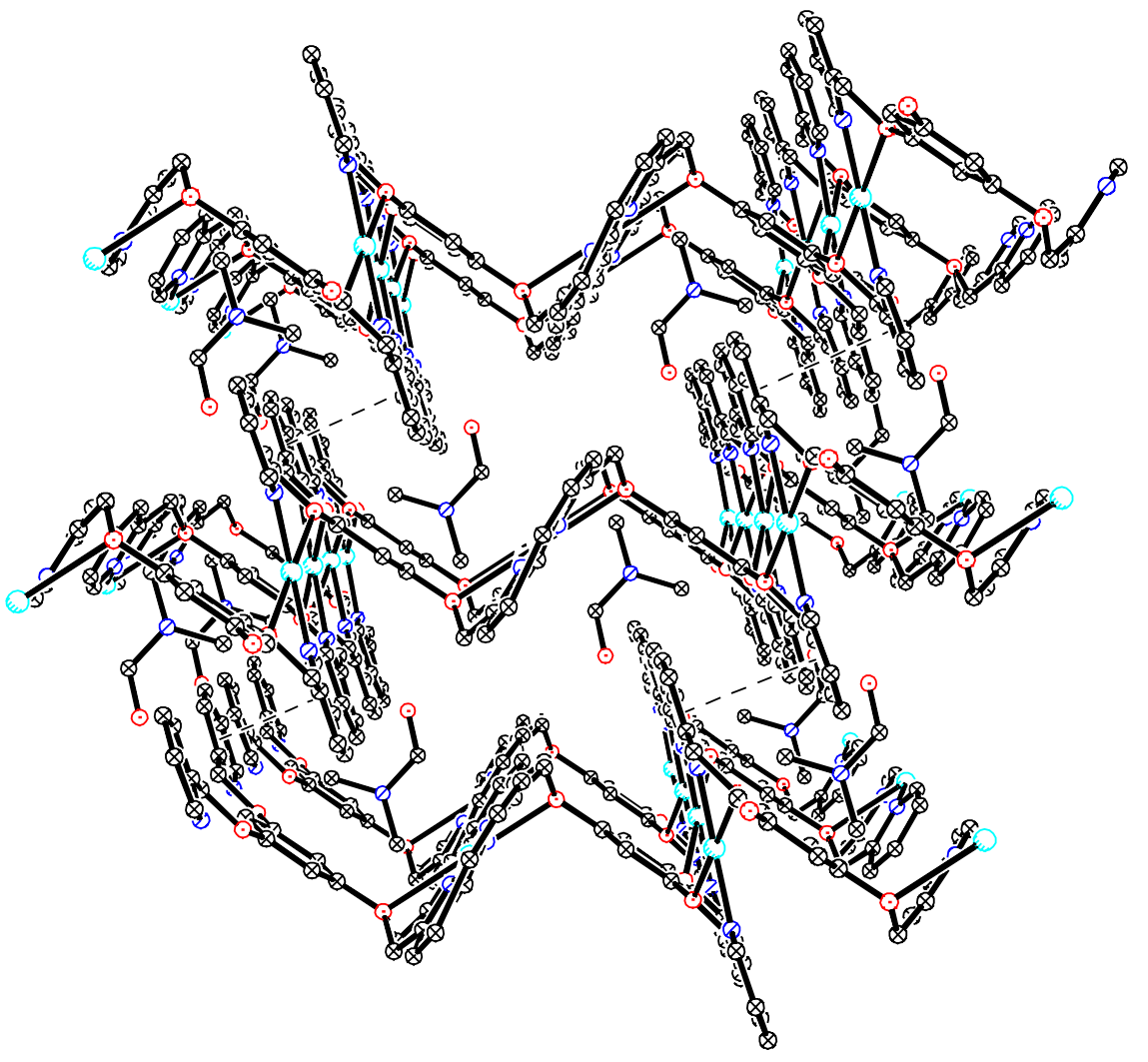

Figure S1 Crystal packing diagram of $\mathbf{3}$ with $\pi-\pi$ interactions indicated by dashed lines. 